\title{
Diversity and Distribution of Order Fabales in Nagpur City, Maharashtra
}

\author{
Pravin U. Dulare ${ }^{1, *}$, Rahul B. Kamble ${ }^{2}$, N. R. Ugemuge ${ }^{3}$, Alka Chaturvedi ${ }^{1}$ \\ ${ }^{1}$ P. G. Department of Botany, University Campus, RTM Nagpur University, Nagpur, India \\ ${ }^{2}$ Department of Botany, Dr. Ambedkar College, Deekshabhoomi, Nagpur, India \\ ${ }^{3}$ Maharashtra State Biodiversity Board, Kadimbagh, Nagpur, India
}

Received June 13, 2020; Revised October 23, 2020; Accepted December 3, 2020

\section{Cite This Paper in the following Citation Styles}

(a): [1] Pravin U. Dulare, Rahul B. Kamble, N.R. Ugemuge, Alka Chaturvedi, "Diversity and Distribution of Order Fabales in Nagpur City, Maharashtra," Advances in Zoology and Botany, Vol. 9, No. 1, pp. 20 - 27, 2021. DOI: 10.13189/azb.2021.090104.

(b): Pravin U. Dulare, Rahul B. Kamble, N.R. Ugemuge, Alka Chaturvedi (2021). Diversity and Distribution of Order Fabales in Nagpur City, Maharashtra. Advances in Zoology and Botany, 9(1), 20 - 27. DOI: 10.13189/azb.2021.090104.

Copyright $\odot 2021$ by authors, all rights reserved. Authors agree that this article remains permanently open access under the terms of the Creative Commons Attribution License 4.0 International License

\begin{abstract}
Order Fabales is one of the largest orders of flowering plants. This order is distributed worldwide and exhibits the greatest diversity of morphological types in tropical and subtropical regions of the world. Order Fabales comprises the major families such as Caesalpiniaceae, Mimosaceae and Fabaceae. To explore the diversity of this order in Nagpur City, regular field visits were made. Present study enumerated the order Fabales with total 59 genera, 126 species, 4 sub-species and 4 varieties of which Family Mimosaceae with 11 genera, 24 species and 2 sub-species. Family Caesalpiniaceae observed with 8 genera, 27 species while the largest family Fabaceae covered most of the diversity in this order with 40 genera, 75 species, 2 sub-species and 4 varieties. The study was documented with flowering and fruiting seasons and distribution in the Nagpur localities.
\end{abstract}

Keywords Order Fabales, Caesalpiniaceae, Mimosaceae, Fabaceae, Nagpur

\section{Introduction}

Biodiversity is the entire diversity of life on the earth. It includes all genes, species and ecosystems. The studies of biodiversity have now assumed greater significance as ecologist and taxonomist try seriously to document the global biodiversity. For this, the exhaustive exploration and taxonomic study i.e. floristic surveys of flora and forest areas have great importance. It is essential to prepare local floras of urban areas where there is severe threat to natural vegetation due to identification of species that are in different stages of vulnerability [1] as well as the various factors that influence the existing vegetation in any region [2].

Nagpur, one of the greenest cities of India, it is also called the 'Garden City of Maharashtra' and is appropriate to and worthy of being the 'Gateway to the Centre Indian Forest. It is also called 'Orange city of India'. One of the most basic tools needs to confront the loss of biodiversity on our planet is lack of floristic information. Floristic studies have acquired increasing importance in recent years in response to the need of developing countries to assess their plant wealth. In the recent years there has been growing interest in plant diversity studies in general and floristic studies in particular. In this connection, regional floristic studies are much importance. It can be achieved by intensive exploration of smaller areas [3].

The Nagpur city is quite rich and varied in its plants composition. In addition to the natural flora of the district, there are large numbers of plants found either in cultivation or introduced for various purposes at one time or other which have now been naturalized in the area [4].

Order Fabales are a climax order achieving the success, which very few other plants groups could reach. Both morphological and chemical features contributed to the success of this group. Of these three families, the 
Mimosaceae evidently are primitive, the Fabaceae, the most advanced and the Caesalpiniaceae, the intermediate taxon. According to Hutchinson [5], order Leguminales is best split into three separate families: Caesalpiniaceae, Mimosaceae and Papilionaceae and treated the 3subfamilies under the order Leguminales. Engler and Prantl [6], Bentham and Hooker [7] and some other taxonomists have considered Caesalpinioideae, Mimosoideae and Papilionoideae as sub-families of the family leguminosae under the order Rosales. These three sub-families are treated as distinct families by many botanists $[8,9]$ except those who cling to tradition. Authors accepting them as separate families treat diversity of fruits and certain other characters. According to Takhtajan [8] and Cronquist [9] order Leguminales changed into order Fabales containing 3 families Mimosaceae, Caesalpiniaceae and Fabaceae.

The Mimosaceae, a tropical or subtropical family mostly of and regions are a comparatively small family of 50 genera containing 3000 or more species. Caesalpiniaceae is a tropical family of about 150 genera and 2200 species. The plants are mostly trees and shrubs and a few herbs. Fabaceae is a large family of 440 genera and about 12000 species. Plants are mostly herbs, undershrubs, shrubs though few trees are also there.

\section{Materials and Methods}

Extensive visits to different parts of Nagpur City categorizing into East Zone, West Zone, North Zone, South Zone and Central Zone including prominent localities like University Campus, Ambazari, Ramnagar, Dharampeth, Laxminagar, NEERI colony, Ganesh tekdi, Pannase Lay-out, Railway station, LIT, Dabha, Ravinagar, Maharajbag, PDKV, Seminary hills, Satpuda Botanical Garden, Telangkheri, Futala, Ramdaspeth, Wardhaman Nagar, Wardha Road, Amravati road, Mahal, Anmol Nagar, Kamptee road, Civil lines etc. During every visit, the specimens were collected for laboratory analysis. All the specimens collected were serially numbered, field notes were recorded in field books with data like habit, habitat, height of the plant, colour of the flowers scent if any, number of individuals and other pertinent features for the purpose of precise identification of the specimens. Each specimen was observed in respect of vegetative, flowering and fruiting condition. Specimens were described; identified and authentified with help of standard literatures, different floras, research papers and reports viz. Flora of Nagpur District [10], Flora of Maharashtra State [11], Flora of Marathwada [12]. The voucher specimens of the collections have been deposited at the Herbarium, PGTD Botany, RTM Nagpur University, Nagpur.

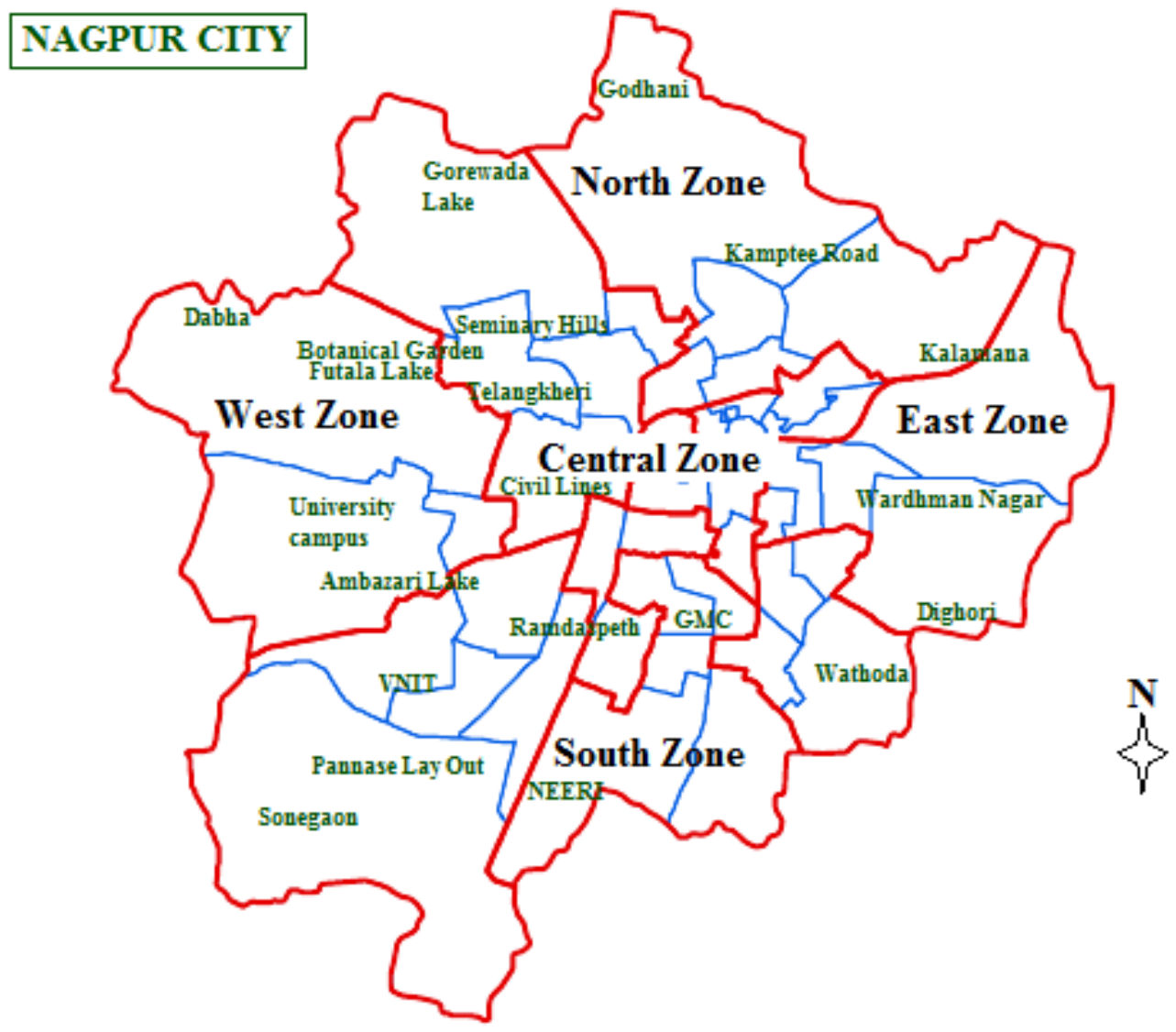

Figure 1. Nagpur City Map showing working zones 


\section{Results and Discussion}

\subsection{Analysis of the Diversity of Order Fabales in Nagpur City}

Present investigation enumerated plants of order Fabales i.e. Family Mimosaceae, Caesalpiniaceae and Fabaceae of Nagpur city with total numbers of 59 genera with 126 species, 4 subspecies and 4 varieties. From which, Mimosaceae comprises 11 genera with 24 species and 2 subspecies (Table No. 2.1). Caesalpiniaceae have an account of 8 genera with 27 species (Table No. 2.2) while Family Fabaceae contributed with maximum numbers of 40 genera with 75 species, 2 subspecies and 4 varieties (Table No. 2.3). Of the three families of order Fabales, the family Fabaceae is dominant and widely distributed in different localities of the city. The present work have compared with the earlier work of Ugemuge [10] and Graham [13]. The frequency percentage in the form of occurrence and distribution of all three families also calculated in co relation with present work's output i.e. genera 59 and species 126 (Table No. 1).

Graham [13] recorded 71 species of order Fabales from Nagpur and Telangkheri farm while Ugemuge [10] documented 105 species belonging to 53 genera from Nagpur city. Panda [14] have recorded the 35 native species of the order Fabales from the Bhadrak District of Odisha, India while our study documented with 126 species.

Table 1. A comparison between status of order Fabales of Nagpur city as per Graham [11] and Ugemuge [8] with present work

\begin{tabular}{|c|c|c|c|c|c|c|c|}
\hline \multirow{2}{*}{ Comparative Analysis } & & \multicolumn{2}{|c|}{ Present Work } & \multicolumn{2}{|l|}{ Ugemuge (1986) } & \multicolumn{2}{|c|}{ Graham (1911) } \\
\hline & & No. & $\%$ & No. & $\%$ & No. & $\%$ \\
\hline \multirow{2}{*}{ Mimosaceae } & Genera & 11 & 18.64 & 11 & 18.64 & 6 & 10.17 \\
\hline & species & 24 & 19.05 & 19 & 15.08 & 10 & 7.94 \\
\hline \multirow{2}{*}{ Caesalpiniaceae } & Genera & 8 & 13.55 & 8 & 13.55 & 3 & 5.08 \\
\hline & species & 27 & 21.43 & 27 & 21.43 & 8 & 6.35 \\
\hline \multirow{2}{*}{ Fabaceae } & Genera & 40 & 67.80 & 34 & 57.63 & 20 & 33.90 \\
\hline & species & 75 & 59.52 & 59 & 46.83 & 53 & 42.06 \\
\hline Total Genera(as per present work) & & 59 & & Total Species (as per present work) & & 126 & \\
\hline
\end{tabular}

Table 2. Elements observed in Order Fables

Table 2.1. Family: Mimosaceae

\begin{tabular}{|c|c|c|c|c|}
\hline Sr.No & Scientific Names & Vernacular name & $\begin{array}{l}\text { Flowering \& } \\
\text { Fruiting }\end{array}$ & Location \\
\hline 1 & Acacia auriculiformis A. Cunn. & Australian babul & June-Jan & Nandanvan, Buildi \\
\hline 2 & Acacia campbelli Arn. & & June-March & University Campus \\
\hline 3 & Acacia catechu (L.F.) Willd. & Khair & July-Dec & Ambazari, Seminary hills \\
\hline 4 & Acacia farnesiana (L.) Willd. & Devbabul & Aug-July & Ambazari, Seminary hills \\
\hline 5 & Acacia ferruginea DC. & Pandharakhair & May-Oct & Campus, Seminary hills \\
\hline 6 & Acacia leucophloea (Roxb.) Willd & Hivar & Aug-Feb & Campus, Seminary hills \\
\hline 7 & Acacia modesta Wall. & -- & Feb.- May & Opposite Veterinary College \\
\hline 8 & $\begin{array}{l}\text { Acacia nilotica (L.) Willd. ssp. } \\
\text { astringens (Schum. \& Thonn) Roberty. }\end{array}$ & Vedibabhul & Aug-Dec & Umrer road, Campus \\
\hline 9 & $\begin{array}{l}\text { Acacia nilotica (L.) Willd. ssp. indica } \\
\text { (Bth.) Brenan. }\end{array}$ & Babhul & June-April & Campus, Telangkheri, Ambazari \\
\hline 10 & Acacia pennata (L.) Willd. & Jari, Chilar & Aug-Jan & Ambazari, Seminari hills \\
\hline 11 & Acacia tomentosa Willd. & Anjar & Dec-Mar & Campus, Seminary hills \\
\hline 12 & Adenanthera pavonina $\mathrm{L}$. & Thorla Gunj & Mar-May & Maharajbag \\
\hline 13 & Albizia lebbeck (L.) Willd. var. lebbeck & Shirish & Feb-Dec & Campus, Ambazari, Seminari hills \\
\hline 14 & Albizia procera (Roxb.) Bth. & Pandhara Shirish & May-Dec & Campus, Wardha road \\
\hline 15 & $\begin{array}{c}\text { Dichrostachys cinera }(\mathrm{L} .) \text { var. indica } \\
\text { Brenan \& Brumit. }\end{array}$ & Sagunkati & Sept-Jan & Kalmeshwar road, Vayusena Nagar \\
\hline 16 & Leucaena latisiliqua (L.) Gills. & Subabhul & $\begin{array}{c}\text { Throughout the } \\
\text { year }\end{array}$ & Campus, Telangkheri, Umrer road \\
\hline 17 & Mimosa hamata Willd. & Chilati & June-Oct & Campus, Seminary hills \\
\hline 18 & Mimosa pudica $\mathrm{L}$. & Lajalu & June-Jan & $\begin{array}{l}\text { PG Boys hostel RTMNU, } \\
\text { Maharajbag }\end{array}$ \\
\hline 19 & Mimosa rubicaulis Lam. & Arai & June-Dec & Kalmeshwarroad, Campus \\
\hline 20 & Neptunia triquetra (Vahl.) Btm. & Lajalu & June-Nov & Campus \\
\hline 21 & Parkia biglandulosa Wight \&Arn. & Chenduphal & Dec-Apr & Boys hostel RTMNU, Campus \\
\hline 22 & Pithecellobium dulce (Roxb.) Btm. & Vilayti Chinch & Jan-June & Campus, Vayusena Nagar \\
\hline 23 & Prosopis cineraria (L.) Druce. & Shami & Dec-June & Mahal, Bharat Nagar \\
\hline 24 & Prosopis juliflora (Swartz.) DC. & -- & Feb-Oct & Amravati road, Kamptee road \\
\hline 25 & Samanea saman (Jacq.) Merr. & Rain tree & Mar-Sept & Bharat Nagar, Wathoda, Campus \\
\hline
\end{tabular}


Table 2.2. Family: Caesalpiniaceae

\begin{tabular}{|c|c|c|c|c|}
\hline 26 & Bauhinia malabarica Roxb. & - & Sept-Feb & VC Bungalow RTMNU \\
\hline 27 & Bauhinia purpurea $\mathrm{L}$. & RaktaKanchan & Sept-Feb & $\begin{array}{l}\text { Telangkheri, Cultivated in } \\
\text { gardens }\end{array}$ \\
\hline 28 & Bauhinia racemosa Lam. & Apta & Apr-Oct & Seminary hills, Ambazari \\
\hline 29 & Bauhinia vahlii Wight \& Arn. & - & Apr-Mar & Campus \\
\hline 30 & Bauhinia variegata $\mathrm{L}$. & Kachnar & Dec-May & Telangkheri \\
\hline 31 & Caesalpinia bonduc (L.) Roxb. & Sagargota & July-Jan & Futala,Umrer road \\
\hline 32 & Caesalpinia corearia (Jacq.) Willd. & Libi-dibi & Sept-Dec & Ambazari \\
\hline 33 & Caesalpinia pulcherrima (L.) Swartz. & Shankasur & Throughout the year & Ambazari, Wardhaman Nagar \\
\hline 34 & Cassia absus $\mathrm{L}$. & Kankuti & Aug-Nov & Amravati road, Campus \\
\hline 35 & C. alata $\mathrm{L}$. & - & Sept-Jan & $\begin{array}{c}\text { Amravati road, Itwari Rail. } \\
\text { Station, Ambazari }\end{array}$ \\
\hline 36 & Cassia bicapsularis $\mathrm{L}$. & - & Sept-Dec & Futala, Telangkheri \\
\hline 37 & Cassia fistula $\mathrm{L}$. & Amaltas & Mar-Oct & $\begin{array}{c}\text { Telangkheri, campus, Shankar } \\
\text { Nagar }\end{array}$ \\
\hline 38 & Cassia javanica $\mathrm{L}$. & - & July-Sept & Maharajbag, Sakkardara \\
\hline 39 & Cassia occidentalis L. & Rantarota & Aug-Mar & Seminary hills \\
\hline 40 & Cassia pumila Lam. & - & Aug-Dec & Campus \\
\hline 41 & Cassia renigera Wall. & - & Apr-June & Sakkardara \\
\hline 42 & Cassia senna $\mathrm{L}$. & Sonamukhi & Throughout the year & Planted in gardens, Maharajbag \\
\hline 43 & Cassia siamea Lam. & - & Aug-Feb & Campus, Bharat Nagar \\
\hline 44 & Cassia sophera $\mathrm{L}$. & - & Aug-Jan & $\begin{array}{l}\text { Telangkheri, Shri. Ayurvedic } \\
\text { College }\end{array}$ \\
\hline 45 & Cassia tora $\mathrm{L}$. & Tarota & July-Nov & Ambazari, Campus \\
\hline 46 & Cassia uniflora Mill. & - & Aug-Dec & Campus, LIT \\
\hline 47 & Delonix elata (L.) Gamble. & Gulmohar & June-Dec & Amravati Road \\
\hline 48 & Delonix regia (Boj. ex Hook) Raf. & Gulmohar & Apr-Oct & Bharat Nagar, Buildi \\
\hline 49 & Hardwickia binata Roxb. & Anjan & July-Mar & Seminary hills, Amravati road \\
\hline 50 & Peltophorum pterocarpum (D.C.) Baker & - & Aug-Feb & Campus, Amravati road \\
\hline 51 & Saraca asoca (Roxb.) de Wilde. & Seeta Ashok & Jan-Apr & Maharajbag \\
\hline 52 & Tamarindus indica $\mathrm{L}$. & Chinch, Imli & Feb-Nov & Telangkheri, Campus, VNIT \\
\hline
\end{tabular}

Table 2.3. Family: Fabaceae

\begin{tabular}{|c|c|c|c|c|}
\hline 53 & Abrus precatoreus $\mathrm{L}$. & Gunj & Sep-Dec & Futala,Campus \\
\hline 54 & Aeschynomene aspera $\mathrm{L}$. & - & Sep-Dec & Campus \\
\hline 55 & Alysicarpus bupleurifolius (L.) DC. & - & Aug-Sep & Campus \\
\hline 56 & Alysicarpus hamosus Edgew. & - & Aug-Oct & Campus, Ambazari. \\
\hline 57 & $\begin{array}{c}\text { Alysicarpus heyneanus Wight \& Arn. var. } \\
\text { heyneanus }\end{array}$ & - & July-Oct & Campus \\
\hline 58 & $\begin{array}{c}\text { Alysicarpus longfolius (Rottl. ex Spreng.) } \\
\text { Wight \& Arn. }\end{array}$ & - & Oct-Feb & Campus, Seminary hills. \\
\hline 59 & Alysicarpus monilifer (L.) DC. & - & July-Oct & Campus \\
\hline 60 & Alysicarpus ovalifolius (Schum.) J.Leon. & - & Aug-Oct & Campus, LIT \\
\hline 61 & $\begin{array}{c}\text { Alysicarpus scariosus (Rottl. ex Spreng.) } \\
\text { Grah. }\end{array}$ & - & Aug-Dec & Campus \\
\hline 62 & $\begin{array}{c}\text { Alysicarpus tetragonolobus Edgew. var. } \\
\text { tetragonolobus }\end{array}$ & - & Aug-Dec & Campus, LIT, NRCC. \\
\hline 63 & $\begin{array}{c}\text { Alysicarpus vaginalis (L.) DC. var. } \\
\text { nummularifolius Miq. }\end{array}$ & - & Aug-Oct & Campus \\
\hline 64 & Arachis hypogaea $\mathrm{L}$. & Bhuimung & Aug-Nov & Campus \\
\hline 65 & $\begin{array}{l}\text { Butea monosperma (Lam.) Taub. var. } \\
\text { monosperma. }\end{array}$ & Palas & Feb-Apr & Campus, Ambazari \\
\hline 66 & Butea superba Roxb. & Palasvel & Feb-Apr & Campus, Ramnagar \\
\hline 67 & Cajanus cajan (L.) Millsp. & Tur & Oct-Feb & Campus, Ambazari. \\
\hline 68 & Cajanus platycarpus (Bth.) Van der Maesen. & Gophanvel & Sep-Dec & Campus \\
\hline 69 & Cajanus scarabaeoides (L.) du-Petit-Thours. & Rantur & Jun-Dec & Campus \\
\hline 70 & Canavalia gladiate (Jacq.) Dc. & - & Oct-Mar & NEERI, Seminary hills. \\
\hline 71 & Cicer arietinum $\mathrm{L}$. & Chana & Oct-Mar & Ambazari road, Umrer road \\
\hline 72 & Clitoria ternatea $\mathrm{L}$. var. pilosula Wall & - & July-Oct & $\begin{array}{c}\text { Satpuda Botanical Garden, } \\
\text { Campus }\end{array}$ \\
\hline
\end{tabular}




\begin{tabular}{|c|c|c|c|c|}
\hline 73 & Clitoria ternatea $\mathrm{L}$. var. ternatea & Gokarni & July-Dec & Campus, LIT. \\
\hline 74 & Crotalaria calycina Schrank. & - & Aug-Nov & Campus \\
\hline 75 & Crotolaria juncea $\mathrm{L}$. & Boru & Aug-Jan & Campus \\
\hline 76 & Crotolaria linifolia $\mathrm{L}$. & - & Sep-Feb & Campus \\
\hline 77 & Cullen corylifolia (L.) Medik. & Bawachi & Aug-Jan & Campus, Umrer road \\
\hline 78 & Cyamopsis tetragonolobus (L.) Taub. & Gawar & July-Dec & Kamptee road garden \\
\hline 79 & Dalbergia latifolia Roxb. & PahariSheesham & Sep-Feb & Campus, Seminary hills \\
\hline 80 & Dalbergia sissoo Roxb. & Sheesham & Mar-Feb & Campus, Seminary hills \\
\hline 81 & Derris scandens (Roxb.) Btm. & Tupbel & Aug-Oct & Sitabuldi \\
\hline 82 & Desmodium dichotomum (Willd.) DC. & Chikta & Oct-Dec & Telangkheri garden, Campus \\
\hline 83 & Desmodium gangeticum (L.) DC. & Salwan & Aug-Nov & Seminary hills, Campus \\
\hline 84 & Desmodium scorpiurus (Sw.) Desv. & - & Oct-Mar & Telangkherigarden, Campus \\
\hline 85 & Desmodium triflorum (L.) DC. & Ranmethi & Aug-Jan & Telangkherigarden, Campus \\
\hline 86 & Erythrina suberosa Roxb. & Pangara & Mar-Sep & Campus, Seminary hills \\
\hline 87 & Erythrina variegata $\mathrm{L}$. & Pangara & Feb-Apr & $\begin{array}{l}\text { Laxminagar square, } \\
\text { Pandharabodi. }\end{array}$ \\
\hline 88 & Gliricidia sepium (Jacq.) Kunth. & - & Feb-Apr & $\begin{array}{l}\text { Ambazari, Pandhrabodi, } \\
\text { Ganeshpeth }\end{array}$ \\
\hline 89 & Glycine $\max (\mathrm{L})$. & Soyabean & Sep-Nov & Umrer road, PDKV. \\
\hline 90 & Goniogyna hirta (Willd.) Ali & - & Sep- Aug-Jan Nov & Ambazari, Pardi \\
\hline 91 & Indigofera astragalina DC. & - & Aug-Oct & Ambazari, Pardi \\
\hline 92 & Indigofera cordifolia Heyne. & - & Aug-Jan & Campus, LIT \\
\hline 93 & Indigofera linifolia (L.) Retz. & - & Aug-Jan & Campus, LIT \\
\hline 94 & Indigofera linnaei Ali. & Bhuiguli & Aug-Jan & Campus,LIT, Wardha road \\
\hline 95 & $\begin{array}{l}\text { Indigofera trifoliata L. var. duthiei (Drumm. } \\
\text { Ex Naik.) Sanj. }\end{array}$ & Sarpot & Aug-Dec & Campus, Amravati Road \\
\hline 96 & Indigofera trita $\mathrm{L}$. var. trita. & - & Aug-Jan & Campus \\
\hline 97 & Lablab purpureus (L.) Sweet. & Popat & Oct-Feb & $\begin{array}{l}\text { Cultivated in garden, } \\
\text { Ravinagar, Telangkheri. }\end{array}$ \\
\hline 98 & $\begin{array}{l}\text { Lablab purpureus (L.) Sweet. var. lignosus } \\
\text { (L.) King. }\end{array}$ & Waal & Oct-Feb & Cultivated in garden, Home \\
\hline 99 & Lathyrus aphaca $\mathrm{L}$. & Ranwatana & Jan-Mar & Seminary hills, Marajbagh \\
\hline 100 & Lathyrus sativus $\mathrm{L}$. & Lakh & Jan-Mar & Umrer road \\
\hline 101 & Lens culinaris Medik. & Masur & Dec-Feb & Mahal, Ambazari \\
\hline 102 & Melilotus alba Medik. & Ranmethi & Feb-Mar & Campus, Sakkardara \\
\hline 103 & Melilotus indica (L.) All. & Ranmethi & Jan-Mar & Campus \\
\hline 104 & Millettia peguensis Ali. & Ranmethi & Feb-Oct & Rare, Campus, Maharajbagh \\
\hline 105 & Mucuna pruriens (L.)DC. & Khajkuiri & Sep-Feb & Campus, Seminary hills \\
\hline 106 & Ougeinia oojeinensis (Roxb.) Hochr. & Kalapals, Tiwas & Feb-Apr & Seminary hills \\
\hline 107 & Phaseolus vulgaris $\mathrm{L}$. & Vilaytiseem & Dec-Mar & Seminary hills, Campus \\
\hline 108 & Pisum sativum L. & Watana & Dec-Mar & Campus, Seminary hills. \\
\hline 109 & Pongamia pinnata (L.) Pierre. & Karanj & Feb-May & Campus, Seminary hills \\
\hline 110 & Pseudarthria viscida (L.) Wt. \&Arn. & Chikta & Aug-Nov & $\begin{array}{l}\text { Satpuda Botanical Garden, } \\
\text { Seminary hills }\end{array}$ \\
\hline 111 & $\begin{array}{c}\text { Pterocarpus marsupium Roxb. var. } \\
\text { marsupium. }\end{array}$ & Bijja & Dec-Mar & Seminary hills. \\
\hline 112 & Rhynchosia bracteata Bth. & - & Oct-Nov & Seminary hills \\
\hline 113 & Rhynchosia minima (L.) DC. & Turel & July-Dec & Campus, Saoner road \\
\hline 114 & Sesbania bispinosa (Jacq.) W. F. Wight. & Ran-Shevra & Sep-Jan & Campus \\
\hline 115 & Sesbania grandiflora (L.) Poir. & Heti & Nov-Mar & LIT, Mahal. \\
\hline 116 & Tephrosia pumila (Lamk.) Pers & - & Aug-Jan & Campus, LIT \\
\hline 117 & Tephrisia purpurea (L.) Pers. & Unhali, Diwali & July-Jan & Campus, Kamptee road \\
\hline 118 & Tephrosia strigosa (Dalz.) Sant & - & Aug-Oct & Ambazari \\
\hline 119 & Tephrosia villosa (L.) Pers. & - & Aug-Oct & Campus, Ambazari \\
\hline 120 & Teramnus labialis (L. f.) Spreng. & Tipani & Aug-Dec & Campus, LIT. \\
\hline 121 & Trigonella foenum-graecum L. & Methi & Feb-Mar & Ambazari, Telangkheri \\
\hline
\end{tabular}




\begin{tabular}{ccccc}
\hline 122 & Uraria picta (Jacq.) Desv. & Pitwan & Sep-Oct & Rare, Gorewada \\
\hline 123 & Vigna angularis (Willd.) Ohwi \& Ohashi. & Udid & Sep-Nov & Telangkheri \\
\hline 124 & Vigna mungo (L.) Hepper. & Moong & Aug-Nov & Campus, Telangkheri. \\
\hline 125 & Vigna radiata (L.) R. & JangliMoong & Aug-Oct & Telangkheri \\
\hline 126 & Vigna trilobata (L.) Verdc. var. trilobata. & Jangli Math & Aug-Oct & Campus, Telangkheri. \\
\hline 127 & $\begin{array}{c}\text { Vigna unguiculata } \text { (L.) Walp. ssp. cylindrica } \\
\text { (L.) van- Eseltin. }\end{array}$ & Chavli & Feb-Oct & Seminary hills, Umrer road \\
\hline $\begin{array}{c}\text { Vigna unguiculata } \text { (L.) Walp. ssp. } \\
\text { unguiculata. }\end{array}$ & Barbati & June-Oct & Telangkheri, Umrer road \\
\hline 129 & Zornia diphylla (L.) Pers. & - & Aug-Oct & Campus, Dabha road \\
\hline 130 & Zornia gibbosa Span. & Thipri & Aug-Oct & Campus, LIT. \\
\hline
\end{tabular}

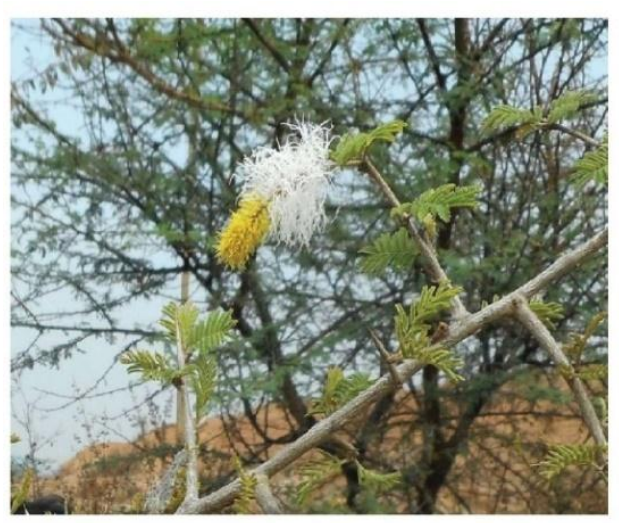

Dichrostachys cinera (L.) var. indica.

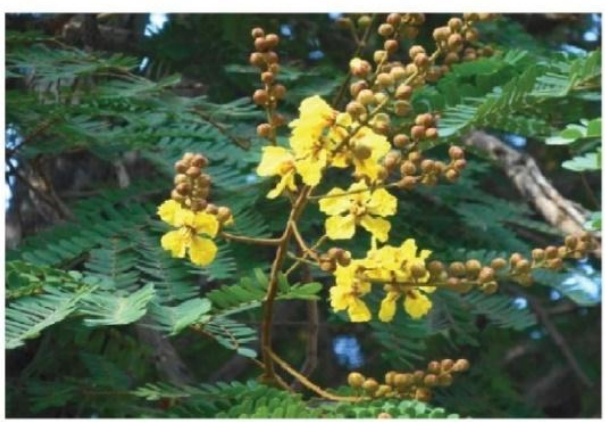

Peltophorum pterocarpum (D.C.) Baker.

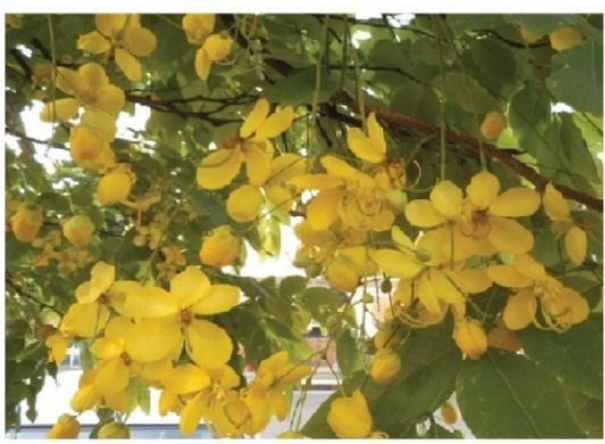

Cassia fistula $\mathrm{L}$.

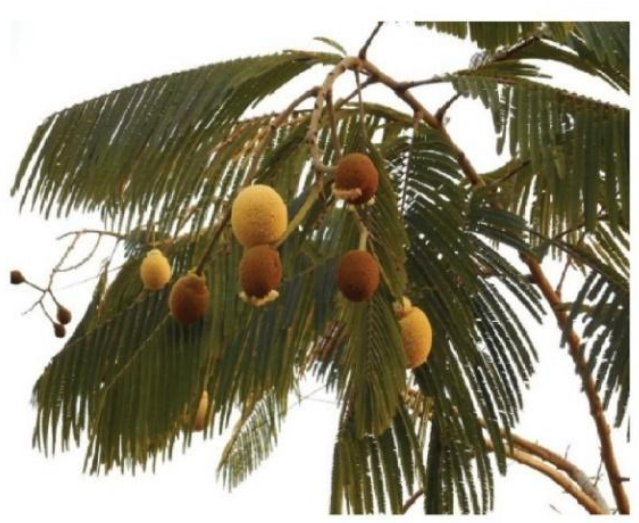

Parkia biglandulosa Wight. \& Arn.

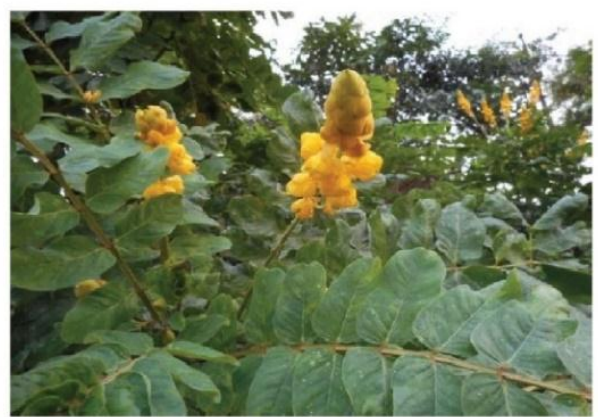

Cassia alata L.

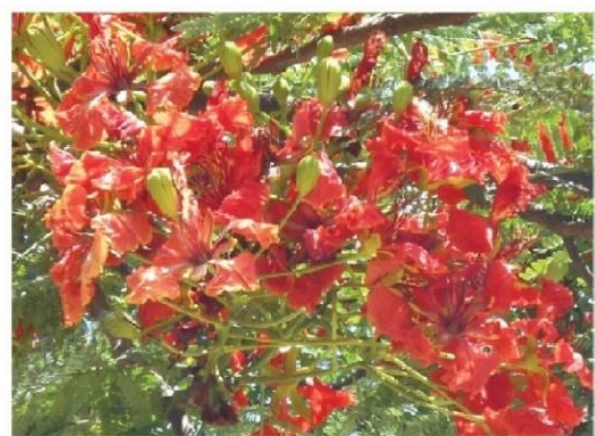

Delonix regia (Boj. ex Hook.) Raf. 


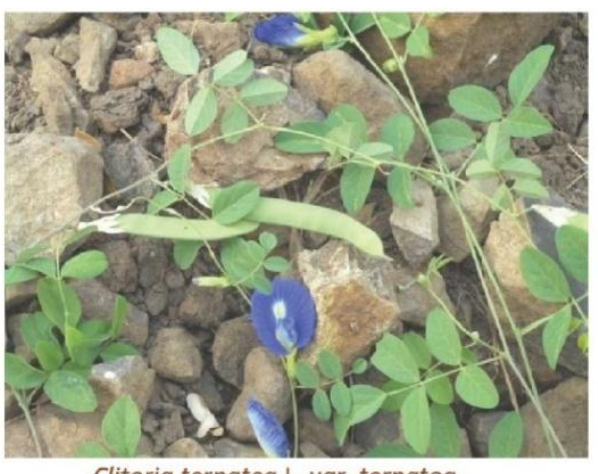

Clitoria ternatea L. var. ternatea.

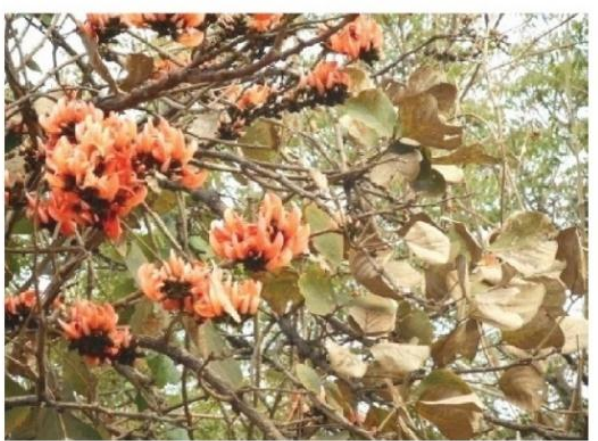

Butea monosperma (Lam.) Taub.

\section{Conclusions}

Present investigation enumerated plants of order Fabales in Nagpur city with potentialities of 59 genera with 125 species, 4 subspecies, 4 varieties belonging to 3 families. From the present study it is concluded that the number of taxa of order Fabales has increased sufficiently to earlier record of Graham [13], Ugemuge [10], Kamble $[15,16]$ and Thakre [17]. It is also pointed out that earlier reported taxa such as Acacia modesta, Uraria picta are found with a single individual in the area, and needs further thorough investigations. So that it can be conserved in the area. Revision of floristic diversity is very important because these studies are considered as the backbone of the assessment of phytodivresity, conservation management and sustainable utilization of natural resources, habit loss and extinction rates of threatened taxa.

\section{REFERENCES}

[1] Padalia H, Chauhan N., Porwal MC, Roy PS. Phytosociological observations on tree species diversity of Andaman Islands, India. Curr. Sci. 87:799-806. 2004.

[2] Parthasarathy N. Tree diversity and distribution in undisturbed and human impacted sites of tropical wet evergreen forest in Southern Western Ghats, India. Biodivers. Conserv. 8:1365-1381. 1999.
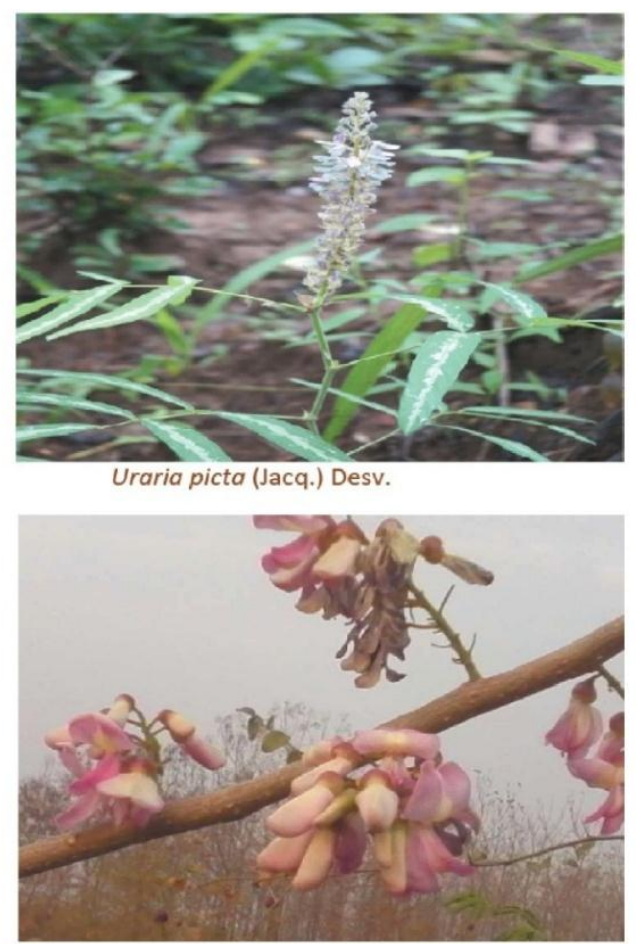

Gliricidia sepium (Jacq.) Kunth.

[3] Kamble RB, Hate S and Chaturvedi A, New additions to the Flora of Nagpur District, Maharashtra. J. New Biol. Rep., 2(1): 09-13.2013-a.

[4] Chaturvedi Arun, Rahul Kamble, N.G. Patil and Alka Chaturvedi. City- Forest relationship in one of greenest city of India. Urban Forestry \& Urban greening Elsevier, 12 (1):79-87.2013.

[5] Hutchinson, J. The families of flowering plants. I. Dicotyledons. MacMillan, London.. 1934

[6] Engler, A. In Engler, A. and K. Prantl: Die natürlichen Pflanzenfamilien, Nachträge1: 69. 1897.

[7] Bentham G and Hooker F. Sistens Dicotyledonum Polypetalarum Ordines XI: Leguminosas-Myrtaceas. Genera Plantarum, 1(2): 433-735.1865.

[8] Takhtajan, A. L. Diversity and classification of flowering plants. Columbia University Press, New York, USA. 1997.

[9] Cronquist, A. An Integrated System of Classification of the Flowering Plants. Columbia Uni. Press, New York. 11011115. 1981.

[10] Ugemuge N.R., Flora of Nagpur District, Shree Publication, Nagpur.1986.

[11] Singh N.P. and Karthikeyan S. Flora of Maharashtra state: Dicotyledones, Vol. I (Ranunculaceae to Rhizophoraceae), Botanical Survey of India. 2000.

[12] Naik, V.N. Flora of Marathwada. Vol. I \& II, Amurt Prakashan, Aurangabad. 1998.

[13] Graham R. J. D. List of wild plant found on the Nagpur and Telangkheri farm. Govt. Press. Nagpur.1911. 
[14] Panda, T., B.K. Pradhan, R.K. Mishra, S.D. Rout \& R.B. Mohanty. Angiosperm diversity in Bhadrak region of Odisha, India. Journal of Threatened Taxa 12(3): 1532615354. 2020.

[15] Kamble Rahul B, Hate Sandeep and Chaturvedi Alka, Some new plant reports to the Flora of Nagpur District, Maharastra - III. Sci. Res. Rep., 3(2): 124- 128.2013-c.

[16] Kamble Rahul B., Sandeep Hate, Arvind Mungole \&
Chaturvedi A., A note on the floristic diversity \&anthropogenic impact in Gorewada International Biopark (proposed) Nagpur, Maharashtra. Proceeding volume of international seminar on 'Multidisciplinary Approaches in Angiosperm systematics' IAAT 2008. In Biodiversity Assessment and conservation: Edited by Maiti \& Mukherjee, vol. 1: Pp: 396-402. (2012).

[17] Thakre MT and Srinivasu T, New (Fabaceae member) records to Nagpur district. M.F.P News, XXII(2):4-5.2012-a. 